\title{
Decellularization of rat aortic valve allografts reduces leaflet destruction and extracellular matrix remodeling
}

\author{
R. W. Grauss, $\mathrm{MSc}^{\mathrm{a}}$ \\ M. G. Hazekamp, MD, PhD \\ S. van Vliet, $\mathrm{MSc}^{\mathrm{a}}$ \\ A. C. Gittenberger-de Groot, $\mathrm{PhD}^{\mathrm{a}}$ \\ M. C. DeRuiter, PhDa
}

From the Departments of Anatomy and Embryology ${ }^{\mathrm{a}}$ and Thoracic Surgery, ${ }^{\mathrm{b}}$ Leiden University Medical Center, Leiden, The Netherlands.

Received for publication March 20, 2003; revisions requested April 21, 2003; revisions received April 29, 2003; accepted for publication June 4, 2003.

Address for reprints: Marco C DeRuiter, $\mathrm{PhD}$, Department of Anatomy and Embryology, Leiden University Medical Center, PO Box 9602, 2300 RC Leiden, The Netherlands. (E-mail: M.C.DeRuiter@LUMC.nl).

J Thorac Cardiovasc Surg 2003;126: 2003-10

Copyright () 2003 by The American Association for Thoracic Surgery

$0022-5223 / 2003 \$ 30.00+0$

doi:10.1016/S0022-5223(03)00956-5
Objectives: Decellularization of aortic valve allografts in advance of transplantation is a promising approach to overcome immune-induced early graft failure. In this study the effects of in vitro cell extraction on extracellular matrix molecules and in vivo remodeling of decellularized aortic valves were investigated in a heterotopic aortic valve rat implantation model.

Methods: Rat aortic valve conduits were decellularized by a 2-step detergentenzymatic extraction method involving sodium dodecyl sulfate in combination with RNase and DNase. Cellular and acellular allogeneic $(2 \times, \mathrm{n}=4)$ and syngeneic valve grafts $(2 \times, \mathrm{n}=3)$ were grafted infrarenally into the descending aorta for 21 days. Immunohistochemical techniques were used to study extracellular matrix constitution (elastin, collagen, fibronectin, and chondroitin sulfate) and cellular infiltration.

Results: The decellularization procedure resulted in a complete loss of all cellular structures from the entire valve conduit with minimal damage to the extracellular matrix. All transplanted cellular allografts became deformed, swollen, and acellular with major changes in extracellular matrix structure. The transplanted decellularized allografts, however, retained normal preserved valve leaflets comparable to transplanted cellular and acellular syngeneic grafts. With the exception of cellular syngeneic grafts, all other grafts showed retrovalvular thrombi.

Conclusions: Damage to the valves caused by decellularization technique is much less than the damage caused by the recipient's immune response. In vitro removal of viable cells in (cryopreserved) homografts may decrease graft failure. Seeding with autologous or major histocompatibility complex-matched donor endothelial cells will be necessary to diminish damage induced by an absent blood-tissue barrier.

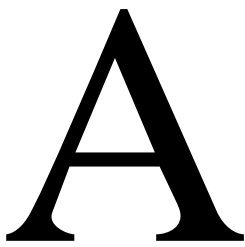

ortic valve allografts (AVA) ultimately fail in the long term, with an effective lifetime around 15 years ${ }^{1}$ in adults and much less in children. ${ }^{2}$ There is evidence that early AVA failure is caused by a donor-specific immune response. ${ }^{3,4}$ Aortic valve transplantation in the rat has shown that AVA are capable of eliciting an immune response that is associated with early graft destruction. ${ }^{5-7}$

The concept of engineering the valve graft before transplantation could be a solution in overcoming valve failure. A promising approach is the use of decellularized AVA. The remaining extracellular matrix (ECM) can then be seeded with autologous cells of the recipient before transplantation in vitro or can be repopulated by recipient's cells in vivo after implantation.

Several methods have been developed to produce completely acellular heart valve tissue matrices using multistep detergent-enzymatic extraction, ${ }^{8}$ Triton de- 
TABLE 1. Decellularization methods

\begin{tabular}{lcc}
\hline Treatment & Concentration & Duration (h) \\
\hline Triton X-100 & $1 \%-5 \%$ & 24 \\
Trypsin & $0.5 \%$ & $0.5-1.5$ \\
Trypsin/Triton X-100 & $0.5 \% / 1 \%-5 \%$ & $0.5-1.5 / 24$ \\
SDS & $0.1 \%-1 \%$ & 24 \\
\hline
\end{tabular}

SDS, Sodium dodecyl sulfate.

tergent, ${ }^{9}$ or trypsin/ethylenediaminetetraacetic acid. ${ }^{10}$ A more recent protocol using sodium dodecyl sulfate (SDS) in the presence of protease inhibitors was successful for aortic valve conduit decellularization, and histological analysis showed that the major structural components seemed to be maintained. ${ }^{11}$ Additional efforts should be given to study the effect of cell removal on different types of ECM molecules and the remodeling of the ECM in the transplanted aortic valve.

Heterotopic aortic valve implantation models in genetically identical rat inbred strains are well described.$^{5-7}$ Such models are essential in studying the effect of cell removal on the in vivo behavior of transplanted aortic valves. In this report we demonstrate that complete rat aortic valve conduits treated with SDS, RNase, and DNase result in a completely acellular graft with minimal remodeling to the ECM.

\section{Materials and Methods \\ Experimental Design}

This study included 4 different groups of animals, which consisted of cellular allogeneic $(n=4)$, acellular allogeneic $(n=4)$, cellular syngeneic $(n=3)$, and acellular syngeneic $(n=3)$ heterotopic aortic valve transplantations. Female inbred WAG-Ry (RT-1\% RyHSD) and Brown Norway (RT-1 $/$ RyHSD) rats 9 to 12 weeks old (Harlan CPB, Horst, The Netherlands) were used as syngeneic and allogeneic donors, respectively. Male inbred WAG-Ry (RT$\left.1^{\mathrm{u}} / \mathrm{RyHSD}\right) 9$ to 12 weeks old were used as recipients. All animals were killed 21 days after the valve transplantation. Four nonimplanted cellular valve conduits were used to study normal valve histology, and an additional set of animals was used to optimize the decellularization method.

All experiments were approved by the committee on animal welfare of the Leiden University Medical Centre (LUMC), The Netherlands. Rats were housed under standard conditions in conventional cages, were given standard diet and water ad libitum, and have received humane care in compliance with the Guide for the Care and Use of Laboratory Animals.

\section{Aortic Valve Implantation}

Donor operation. Animals were killed by cervical translocation, after which a median sternotomy was performed followed by en bloc removal of the lungs. The innominate artery, left common carotid artery, and left subclavian artery were dissected and ligated with 8-0 nylon sutures (Ethicon, Johnson \& Johnson Intl, Brussels,
Belgium), as well as both coronary arteries, while intermittently moistening the tissue with Hanks balanced salt solution (HBSS) under sterile conditions. The aortic valve conduit consists of the aortic root, ascending aorta, aortic arch, and descending aorta. A small rim of left ventricular outflow tract myocardium was dissected free. The major part of the tunica adventitia was removed. The conduits were then stored in heparinized buffered saline (12.5 $\mathrm{U} / \mathrm{mL}$ ) on ice or decellularized as described below.

Decellularization of aortic valve conduits. Various decellularization protocols were tested for cell extraction capacity of rat aortic valves (Table 1). First a Triton X-100 decellularization method $^{12}$ was used with varying detergent concentrations $(1 \%$ $5 \%$ ). A second enzymatic extraction method using $0.05 \%$ trypsin was used mainly as previously described ${ }^{10}$ but with extended washing steps (0.5-1.5 hours). Thirdly, a combination of these 2 protocols consisting of a $0.05 \%$ trypsin treatment followed by a 24-hour Triton X-100 treatment was tested. The fourth method was based on a $0.1 \%$ SDS cell extraction method, ${ }^{13}$ to which a DNase and RNase step was added. After the valves were treated for 24 hours at $4^{\circ} \mathrm{C}$ with $0.1 \%$ SDS in distilled water, they were placed in a solution containing RNase A $(20 \mu \mathrm{g} / \mathrm{mL})$ and DNase $(0.2 \mathrm{mg} /$ $\mathrm{mL}$ ) for 1 hour in HBSS at room temperature, followed by washing with HBSS at $4^{\circ} \mathrm{C}$ for 48 hours to remove residual substances. All steps were conducted under continuous shaking (600 rpm). Pending the transplantation the grafts were placed in a sterile heparinized saline solution for maximally 5 hours at $4^{\circ} \mathrm{C}$.

Recipient procedure. The heterotopic aortic valve transplantation described by Oei and colleagues ${ }^{7}$ was slightly modified. In short the recipient rats were anaesthetized with halothane administered by a vaporizer with a waste gas scavenging system in combination with $\mathrm{NO}_{2} / \mathrm{O}_{2}$ in a $50: 50$ or $60: 40$ ratio. A vapor concentration of $3 \%$ to $4 \%$ was used for induction, and anesthesia was maintained with a $0.75 \%$ to $1.5 \%$ halothane concentration. A midline laparotomy was performed, the intestines were mobilized to the right, and the aorta was dissected away from the vena cava from the level of the renal arteries to the aortic bifurcation and crossclamped with a Lee clamp. Within this segment 2 incisions were made to create the distal and proximal openings for the anastomoses. The anastomoses were made, within 50 minutes after clamping, with a continuous 10-0 suture (Ethicon), flushing intermittently with heparinized buffered saline solution $(12.5 \mathrm{U} / \mathrm{mL})$. After 21 days the explanted U-shaped aortic valve conduit was cut a few millimeters above the annulus and the remaining piece was split longitudinally into 3 valve cusp-containing symmetric rectangular pieces.

\section{Histology}

All the valve tissues were fixed overnight in $2 \%$ acetic acid in $100 \%$ ethanol or in $4 \%$ paraformaldehyde, dehydrated in graded ethanol and xylene, and subsequently embedded in paraffin. Serial sagittal sections of $5 \mu \mathrm{m}$ were cut and mounted onto egg white/ glycerine-coated glass slides for histological and immunohistochemical analysis. Parallel sections were routinely stained with hematoxylin-eosin. Resorcin-fuchsin staining was used to visualize elastic laminae. Sirius red was used to detect collagen and Alcian blue to detect glycosaminoglycans (GAG). 


\section{Immunohistochemistry}

Smooth muscle cells were visualized with the 1A4 mouse antihuman antibody recognizing $\alpha$-smooth muscle actin (A2547, Sigma, St Louis, Mo). Extracellular matrix proteins were detected using a mouse anti-chicken chondroitin sulfate (6505, Bio-Yeda, Israel) monoclonal and a rabbit anti-human fibronectin (A245, Dako, Denmark) polyclonal antibody.

Sections were deparaffinated and dehydrated in xylene and graded ethanol and treated with $0.3 \% \mathrm{H}_{2} \mathrm{O}_{2}$ in phosphate-buffered saline solution (PBS; 10 minutes) to quench endogenous peroxidase activity. Thereafter the sections were washed twice in PBS and once in PBS containing $0.05 \%$ Tween-20. Routine immunostaining was performed by overnight incubation with the primary antibodies 1A4 (1:2000), anti-chondroitin sulfate (1:200), and antifibronectin (1:400), diluted in PBS containing $0.05 \%$ Tween and $1 \%$ bovine serum albumin (BSA, Sigma). Control sections were incubated with PBS. After overnight incubation, sections were again washed twice in PBS and once in PBS with $0.05 \%$ Tween20. To detect bound antibodies the sections were incubated with horseradish peroxidase-conjugated swine anti-rabbit (1:250, Dakopatts, Glostrup, Denmark) or rabbit anti-mouse antibody (1:250, Dako) diluted in PBS, containing $1 \%$ BSA and $0.05 \%$ Tween, for 3 hours at room temperature. Sections were washed again and bound immunocomplexes were visualized by treatment with $0.04 \%$ diaminobenzidine tetrahydrochloride and $0.06 \% \mathrm{H}_{2} \mathrm{O}_{2}$ in $0.05 \mathrm{~mol} / \mathrm{L}$ Tris-maleate buffer $(\mathrm{pH} \mathrm{7.6)}$ for 8 minutes at room temperature. Sections were counterstained with hematoxylin for 10 seconds, dehydrated in graded ethanol, and mounted in Entellan (Merck, Darmstadt, Germany).

\section{Results}

\section{Histology of Normal and Decellularized Valves}

Normal valve. Rat aortic valve leaflets consist of 5 to 6 cell layers and do not display a typical 3-layered structure (Figure 1, $a$ ) as is seen in human and porcine aortic valves. The mesenchymal cells in the leaflets do not express $\alpha$-smooth muscle cell (SMC)-specific actin. A small amount of elastin is found in the valve leaflet. At the basis of the valve 4 to 5 lamellae are observed (Figure 1, b), and at the ventricular side of the leaflet only 1 very thin and mostly incomplete lamella of elastin is present. One of the major components of the ECM of the rat aortic valve leaflet is collagen, comprising approximately three quarters of the leaflet (Figure 1,c) predominantly at the arterial side. GAGs, chondroitin sulfate (CS), and fibronectin (FN) are found at the basis of the valve and at the ventricular and arterial side of the leaflet (Figure 1, $d, e, f$ ).

Decellularized valve. Treatment of the valves with a $1 \%$ Triton X-100 solution for a period of 24 hours showed no change in cellularity compared with a normal rat aortic valve (Figure 2, a). Increasing the concentration to $5 \%$ resulted into only a slight decrease in valve cells (Figure 2, $b$ ), with the SMCs not being removed from the aortic wall. The $0.05 \%$ trypsin treatment resulted in a complete loss of valve cells (Figure 2,c), but cells in the media of the aortic wall could not be removed. Combination of the Triton

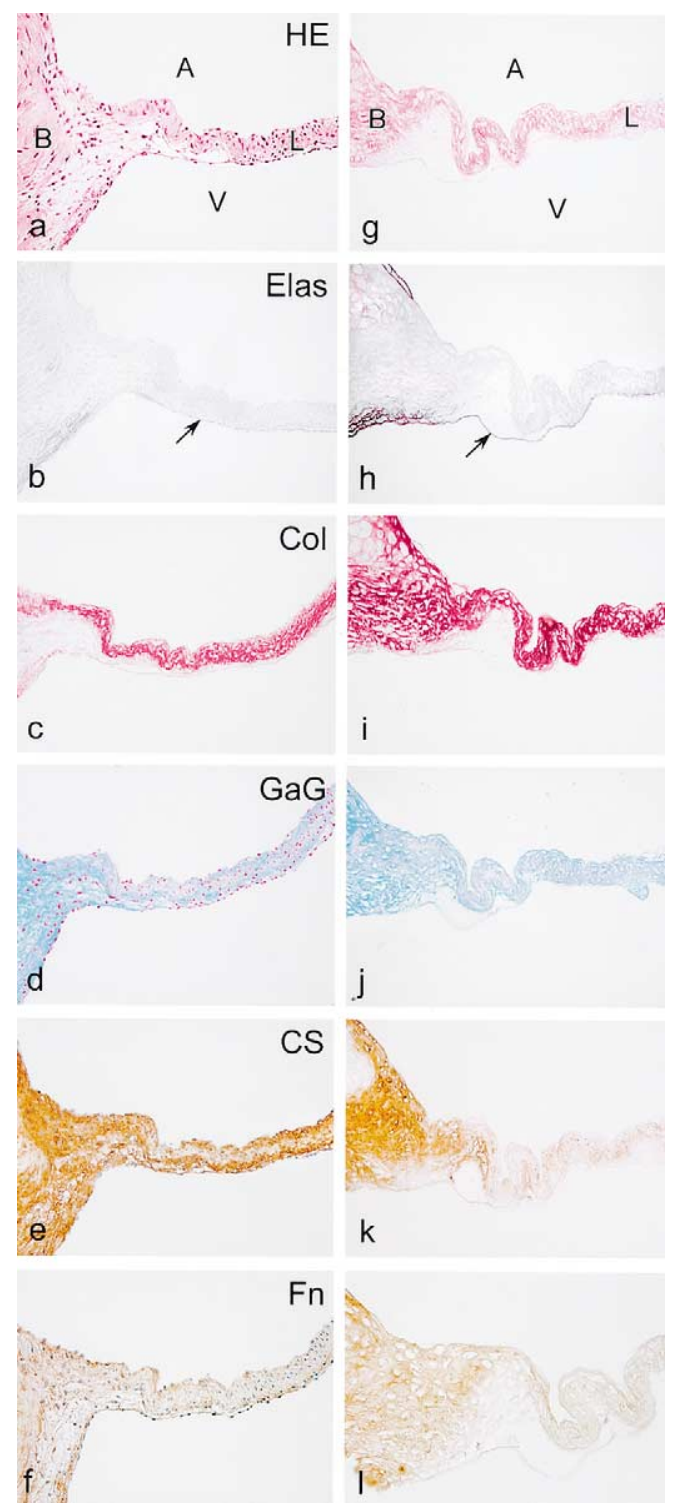

Figure 1. a to I, Histology of a normal adult rat aortic valve (left panel). The valve leaflets consist of 5 to 6 cell layers (a) containing only a small amount of elastin (Elas; b). The ECM is mainly composed of thick layers of collagen ( $\mathrm{Col}$; c), while glycosaminoglycans ( $G a G$; d) as chondroitin sulfate (CS; e) and fibronectin ( $F N$; f) are abundantly expressed. The aortic valve after decellularization using $0.1 \%$ SDS with DNase and RNase (right panel) shows removal of all cellular components $(g)$ while the elastin and collagen fiber distribution were not affected $(h, i)$. The single elastic lamella in the leaflet is indicated by an arrow. Only a considerable washout of CS was seen for the GAG $(j, k, I)$. Arterial $(A)$ and ventricular $(V)$ side of the valve; valve basis $(B)$; valve leaflet (L); hematoxylin-eosin (HE).

$\mathrm{X}-100$ and trypsin protocols resulted in complete decellularization of the entire conduit. However, analysis of the decellularization-induced ECM damage (Figure 2, d) 

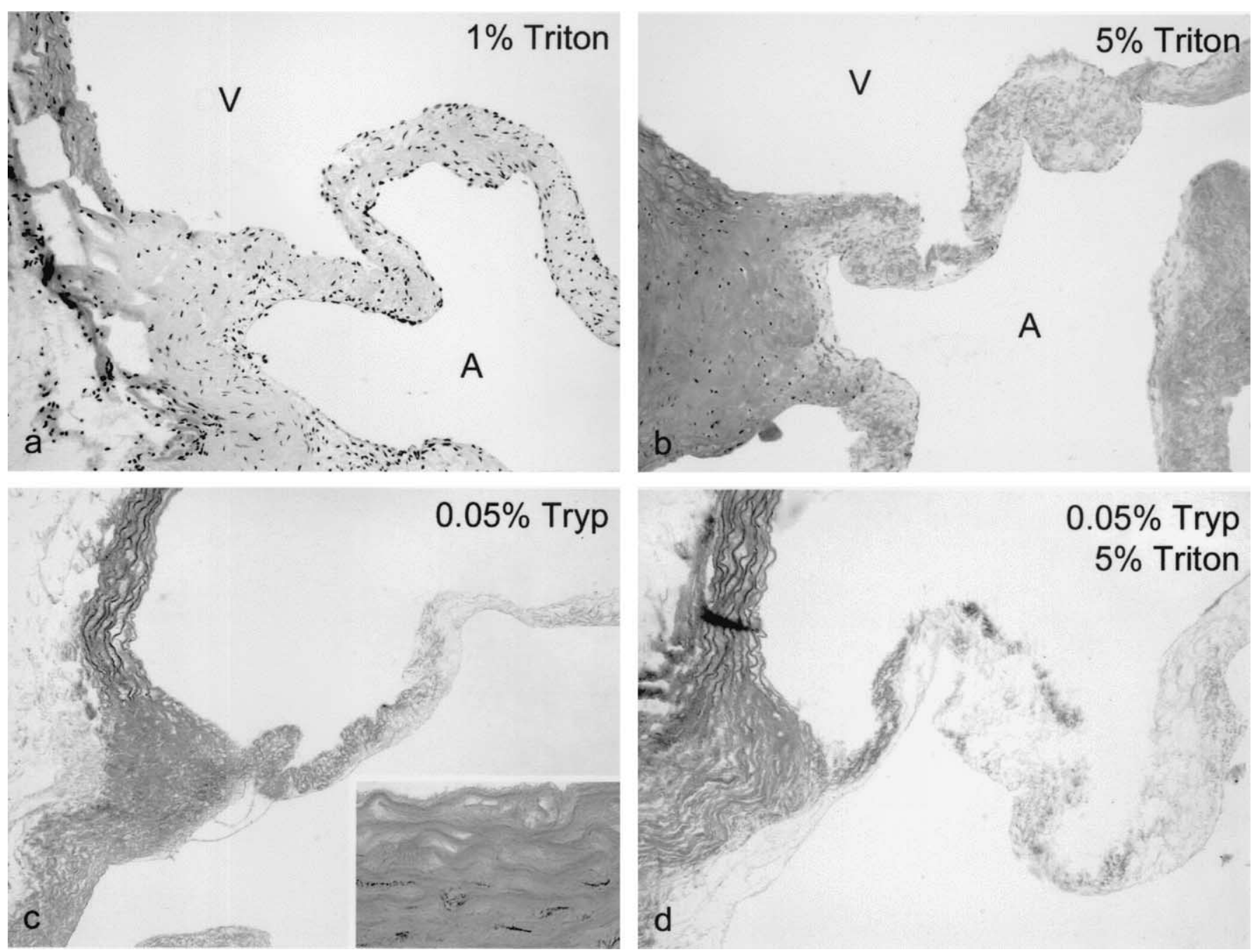

Figure 2. a to d, Various described methods tested to decellularize aortic valves did not remove the cells at all from the valve in the rat (a) or remnants of cells were found at the basis of the leaflet (b) or in the aortic wall (inset in c). Severe damage in leaflets was seen in protocols comprising trypsin (c, d). Arterial (A) and ventricular (V) side of the valve; hematoxylin-eosin staining (HE); Trypsin (Tryp).

showed an almost complete loss of elastin, GAGs including CS, a disruption of the collagen fibers, and a decrease in FN. Furthermore, all protocols comprising trypsin resulted in a severely damaged myocardial rim and aortic wall, making it impossible to create stable suture lines for the anastomosis.

For this reason, we introduced a fourth decellularization method based on SDS. SDS removes all the cells from the myocardial rim, aortic valve, and aorta. However, many acellular basophilic nuclear residues were not washed out from the tissue. To remove these remnants we added DNase and RNase. Histological analysis of valves revealed that this procedure resulted into a complete loss of cellular structures across the complete thickness of the valve leaflet (Figure 1, $g$ ). There was no loss or disruption of the elastic lamellae (Figure 1, $h$ ), and also the collagen distribution appeared to have been preserved (Figure 1,i). Alcian blue staining showed no major changes in GAG distribution after decellularization treatment (Figure $1, j$ ). Immunohistochemical staining, however, showed that the decellularization treatment had resulted in a considerable washout of CS and FN throughout the complete leaflet but was still abundantly present in the basis of the valve (Figure $1, k, l$ ).

\section{Aortic Valve Transplantations}

Cellular allogeneic grafts. In all grafts retrovalvular thrombi were seen in the aortic sinuses (Table 2). Like the syngeneic control transplantations (see below) all grafts showed little to moderate intimal proliferation (Figure 3, $a$, $b$ ). These intimal proliferations were positive for SMC-actin (not shown), collagen, GAG, CS, and FN (Figure 3, $c, d, e$ ). 
TABLE 2. Histological and morphological evaluation of transplanted valves

\begin{tabular}{|c|c|c|c|c|c|c|c|}
\hline & \multirow[b]{2}{*}{ Retro valvular thrombus } & \multirow[b]{2}{*}{ Preserved leaflets } & \multicolumn{5}{|c|}{ Matrix } \\
\hline & & & Elastin & Collagen & GAG & FN & CS \\
\hline \multicolumn{8}{|c|}{ Cellular syngeneic } \\
\hline 1 & No & Yes & $=$ & $=$ & $=$ & $=$ & $=$ \\
\hline 2 & No & Yes & $=$ & $=$ & $=$ & $=$ & $=$ \\
\hline 3 & No & Yes & $=$ & $=$ & $=$ & $=$ & $=$ \\
\hline \multicolumn{8}{|c|}{ Acellular syngeneic } \\
\hline 1 & Yes & Yes & $=$ & $=$ & $=$ & $=$ & $=$ \\
\hline 2 & Yes & Yes & $=$ & $=$ & $=$ & $\downarrow$ & $\downarrow \downarrow$ \\
\hline 3 & Yes & Yes & $=$ & $=$ & $=$ & $\downarrow$ & $\downarrow$ \\
\hline \multicolumn{8}{|c|}{ Cellular allogeneic } \\
\hline 1 & Yes & Deformed & $=$ & $\downarrow \downarrow$ & $=$ & $\downarrow$ & $\downarrow$ \\
\hline 2 & Yes & Deformed & $\downarrow$ & $\downarrow$ & $=$ & $=$ & $\downarrow$ \\
\hline 3 & Yes & Deformed & $\downarrow$ & $\downarrow$ & $=$ & $\downarrow$ & $\downarrow \downarrow$ \\
\hline 4 & Yes & Deformed & $\downarrow$ & $\downarrow$ & $=$ & $\downarrow$ & $\downarrow$ \\
\hline \multicolumn{8}{|c|}{ Acellular allogeneic } \\
\hline 1 & Yes & Yes & $=$ & $=$ & $=$ & $\downarrow$ & $\Downarrow$ \\
\hline 2 & Yes & Yes & $=$ & $=$ & $=$ & $=$ & $\Downarrow$ \\
\hline 3 & Yes & Yes & $=$ & $=$ & $=$ & $\downarrow$ & $\downarrow$ \\
\hline 4 & Yes & Yes & $=$ & $=$ & $=$ & $\downarrow$ & $\downarrow$ \\
\hline
\end{tabular}

Extracellular matrix constitution (glycosaminoglycans, GAG; fibronectin, $F N$; and chondroitin sulfate, $C S$ ) was compared to fresh untransplanted valve leaflets as similar $(=)$, slight decrease $(\downarrow)$, or considerable decrease $(\downarrow)$.

The allogeneic valve leaflets were deformed and swollen while the cells had disappeared (Figure 3, a, b). Major changes in ECM structure, as indicated by a disruption and fragmentation of the elastic lamina at the ventricular side, was seen in all these allografts. The collagen fibers were less compact (Figure 3,c) and there was a decrease of CS (Figure 3,d). No loss of GAG was observed in the valve leaflets. Furthermore, a slight decrease of FN was found in 3 grafts (Figure 3,e) and all grafts were covered with a thin layer of fibronectin.

Acellular allogeneic grafts. All allogeneic acellular grafts contained retrovalvular thrombi. The valve leaflets showed normal morphology (Figure 3, $f, g$ ) with an intact elastic lamina on the ventricular side and regular collagen (Figure $3, h$ ) and GAG distributions. In 2 of 4 grafts intimal proliferation at the ventricular side was observed. A similar decrease in CS expression was found as compared with a nontransplanted, treated valve (compare Figure $3, i$, and Figure $1, k)$. There was also a slight decrease of FN expression in 3 of 4 grafts (compare Figure 3, $j$, and Figure 1, $l$ ). Most valves were covered by a thin layer of FN. Ingrowth of $\alpha$-smooth muscle actin-positive cells at the base of the leaflet was observed (Figure 4,a).

Acellular syngeneic grafts. All acellular syngeneic grafts contained retrovalvular thrombi and showed moderate to severe intimal proliferations extending from the proximal anastomoses to the ventricular side of the valve. The intimal proliferations were positive for SMC-actin (Figure 4, $a$ ), collagen, GAG, CS (Figure 4, b), and FN (Figure 4,c). In all 3 grafts proliferating intimal cells had migrated into the valve leaflet. In most leaflets only the basis of the valve was populated by recipients' cells, but in 1 valve leaflet almost three quarters of the leaflet was repopulated (Figure 4, a). All valve leaflets showed normal morphology with intact elastic lamellae on the ventricular side and regular collagen and GAG distributions. Both the CS and FN content was similar as in the acellular allogeneic group, but in the leaflet that was infiltrated by proliferating intimal cells expression was similar to a fresh leaflet (Figure 4,b,c).

Cellular syngeneic grafts. None of these control grafts were affected by thrombosis in the valve sinuses. All grafts showed moderate to severe intimal proliferation extending from the proximal anastomoses to the ventricular side of the valve and sometimes even extending over the entire valve leaflet. The intimal proliferations stained positive for antiSMC actin, collagen, GAGs, CS, and fibronectin. Except for these surgically induced intimal proliferations the valves did not show any difference in morphology and matrix constitution as compared with a nontransplanted aortic valve.

\section{Aortic Wall}

Decellularization of the aorta using SDS in combination with DNase/RNase resulted in a complete loss of cellular structures from both the media and adventitia. The tunica media became more compact with smaller interlamellar spaces after decellularization (Figure $4, d, e$ ). The collagen distribution in the adventitia was not changed after treatment. GAG distribution showed a more compact appearance similar to that of the elastic lamellae. There was almost no decrease in CS and FN expression. 


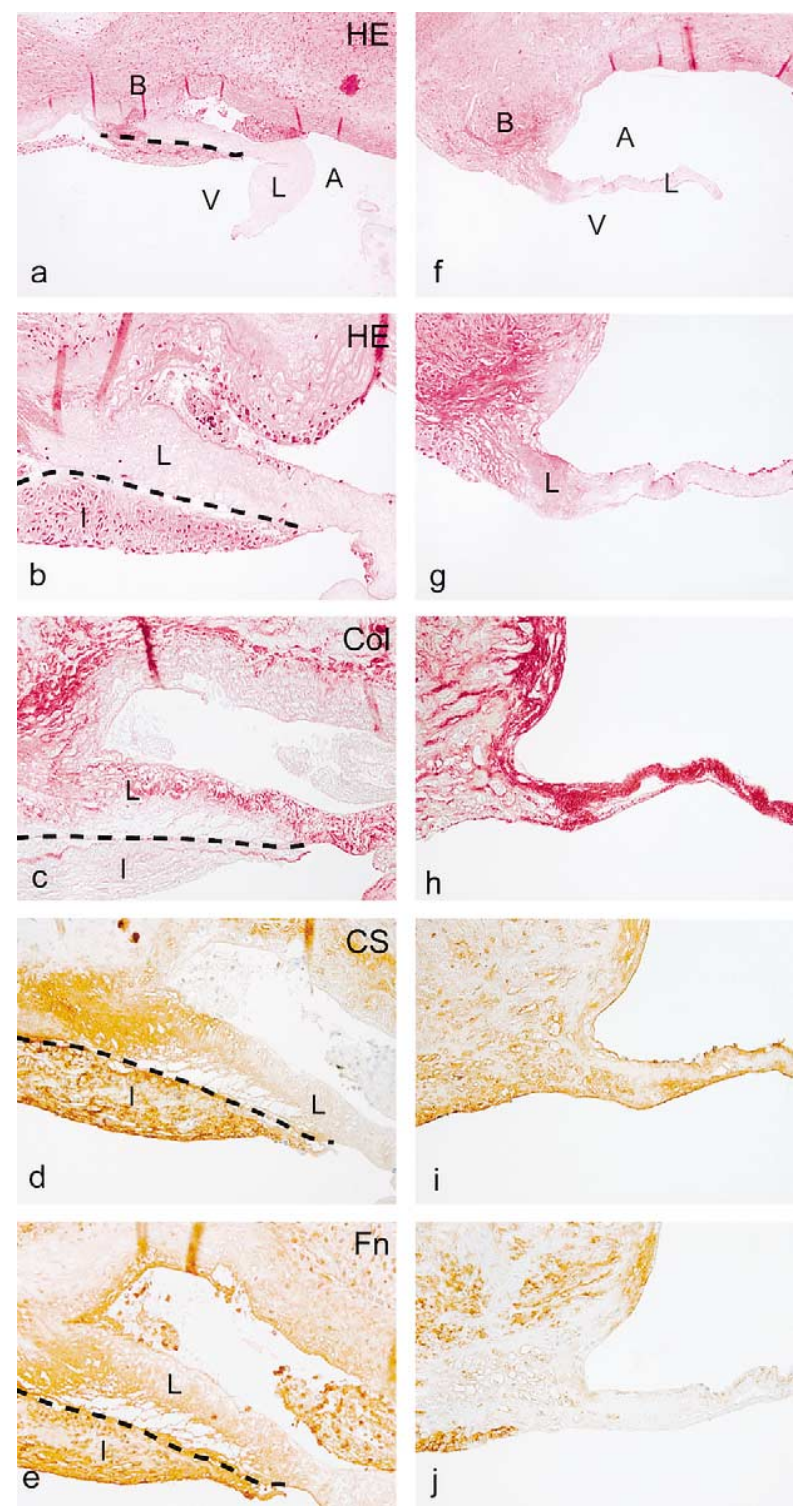

Figure 3. a to $j$, The valve leaflets of transplanted cellular allogeneic aortic conduits (left panel) were deformed and swollen while the cells had disappeared $(a, b)$. The collagen fibers were less compact (Col; c) and there was a decrease of chondroitin sulfate (CS; d). Transanastomotic intimal proliferations (I) were positive for CS and fibronectin ( $F N$; e). Transplanted acellular allogeneic grafts (right panel) demonstrated normal valve leaflets $(f, g)$ with regular collagen (h) and glycosaminoglycans distributions $(i, j)$. Arterial $(A)$ and ventricular $(V)$ side of the valve; valve basis $(B)$; valve leaflet (L); hematoxilin-eosin (HE).

A complete loss of medial SMCs in the aortic root was found in transplanted cellular allogeneic conduits. The loss of SMC was accompanied by a decrease in GAG and CS, and a multifocal disruption of the elastin fibers (Figure 4, $f$ ). In transplanted acellular allogeneic conduits, however, the medial elastic fibers were normally arranged (Figure 4, g) and also the amount of collagen, GAG, and FN in the media did not differ from a nontransplanted acellular aortic conduit. Only a decrease in medial CS expression as compared with a nontransplanted, treated valve conduit was found. The media was still acellular and negative for anti-SMC actin.

The matrix constitution in the controls (acellular syngeneic) did not differ from the acellular allogeneic group. The media was also still acellular and negative for anti-SMC actin. There was no loss or disruption of elastic fibers and also the amount of collagen, GAG, and FN was unchanged.

\section{Discussion}

Extraction of cellular components from heart valve allografts is believed to minimize the immunologically induced structural changes leading to graft failure. Several authors have developed decellularization techniques followed by in vitro reseeding with autologous cells ${ }^{10,14}$ or allowing ingrowth of host cells into the graft after transplantation. ${ }^{8,10,15,16}$ The effect of cell extraction on different types of ECM proteins, however, and the reconstitution of ECM and valve cells after in vivo implantation have not been studied in detail yet. To answer these questions we have chosen to analyze the effect of decellularization on heart valve conduits in rat inbred strains, which are genetically well described and have provided important insights into the role of the immune response in allograft heart valve failure. .,6,17 $^{-17}$

In the present study we developed a 2-step detergentenzymatic extraction method involving SDS in combination with RNase and DNase to produce completely acellular rat aortic heart valve conduits suitable for implantation. Against our expectations previously described methods to decellularize larger canine, ${ }^{8}$ porcine, ${ }^{9}$ and human ${ }^{10,14}$ aortic valves failed in removing cells from the small and compact rat aortic valves. Procedures to detach cells from their surrounding matrix inevitably implies a certain amount of damage to the ECM. Each of the tested decellularization methods resulted in ECM damage. The SDS/RNase/DNase method appeared to be the mildest treatment. It showed no loss or disruption of the major structural components as elastin and collagen. Only a loss of CS and FN in the valve leaflet and a minor loss in the aortic wall was observed. The media of the decellularized aortic wall was more compact and thinner than an untreated aortic wall but is consistent with earlier findings. ${ }^{13}$ In the rat aortic valve transplantation model the morphology and ECM constitution of the decellularized allograft conduits contrasted with the findings in the cellular allograft conduits used as controls. Although the number of animals per group was small, all the explanted cellular allograft leaflets were deformed and swollen, with severe damage to the elastin and collagen fibers accompanied by a loss of CS. The morphology and ECM constitution 


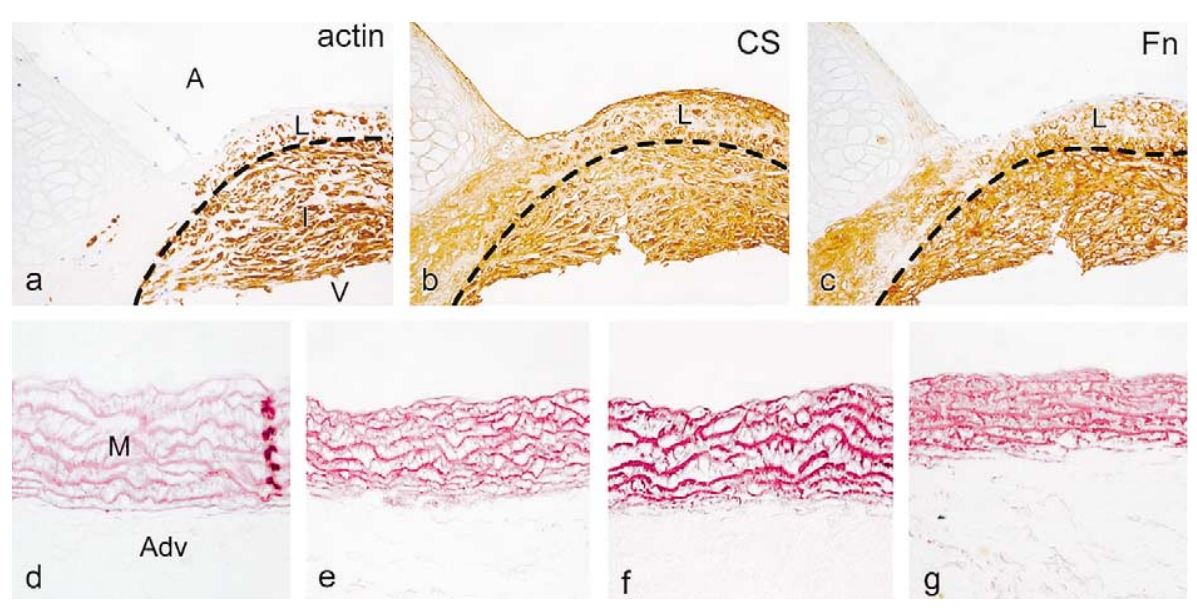

Figure 4. a to $\mathrm{g}$, Several cases were seen with transanastomotic intimal proliferations (upper panel). In case of intimal proliferation actin-positive intimal cells $(I$, a) expressing chondroitin sulfate (CS; b) and fibronectin (FN; c) migrated into the acellular valve leaflet. Allogeneic acellular transplantation: arterial (A) and ventricular (V) side of the valve; valve leaflet (L). The lower panel shows a normal untreated (d), decellularized (e), transplanted allogeneic cellular (f) and a transplanted allogeneic acellular (g) aortic wall. Decellularization resulted in a complete loss of cellular structures from both the media and adventitia (Adv), but the tunica media (M) became more compact with smaller interlamellar spaces $(e, g)$. Multifocal disruption of elastin laminae was often seen in transplanted cellular allogeneic conduits (f). No loss or disruption of elastic fibers could be demonstrated in the acellular allogeneic group, although the media had a more compact appearance.

of the decellularized allograft conduits, however, appeared almost normal. Furthermore, there was a complete loss of valve cells in the cellular allogeneic group, which was not observed in the cellular syngeneic group. This almost complete loss of valve cells was accompanied by loss of ECM structure, which is consistent with findings in allogeneic transplantations in other rat aortic valve transplantation studies. ${ }^{6,17}$

Transplanted cryopreserved homografts rapidly lose their cellular components during the first year of implantation $^{18}$ while the normal tissue architecture is damaged. ${ }^{19}$ Despite an increasing amount of evidence that aortic valve allografts are capable of eliciting a cellular and humoral immune response, ${ }^{4,20-22}$ it is not yet clear what exact role the immune response plays in ECM damage of homografts. A direct relationship between recipient immune activation and structural valve failure is still not proven. An important reason is the impossibility of having valvular biopsies in the clinical setting. Immune suppression or human leukocyte antigen matching for these reasons is applied almost nowhere in case of aortic valve allograft transplantations. After transplantation indicators of immune-mediated injury as persistent up-regulation of leukocyte adhesion molecules $^{23}$ and the presence of neutrophyl granulocytes and depositions of antibodies and complement ${ }^{24}$ are missing. We should bear in mind that these studies have missed the immune response in an earlier phase after implantation.

$\mathrm{Oei}^{25,26}$ demonstrated in his MHC-mismatched rat valve transplantation model an infiltration of recipient $\mathrm{CD} 4+$,
$\mathrm{CD} 8+$, and CD68 + $\mathrm{T}$ cells and allogeneic antigen-presenting denditric cells (MHC class II) in the graft within 7 days after transplantation. Influx of macrophages by local expression of cytokines and chemokines from the activated T cells led to complete loss of stromal cells and distorted leaflet structure at day 21. In the present study we demonstrated in the same model that removal of the allogeneic MHC class Iand II-expressing cells and cell debris in advance of the transplantation prevents the immune-induced damage that results from influx of lymphoid cells. Even in MHCmatched valve allograft transplantations it can be argued that decellularization of the valves will have a positive effect on the survival and functionality of the valve in that the dead cells caused by, for example, cryopreservation, warm ischemia time, and/or mechanical damage also induce a T-cell-mediated macrophage influx.

A complication in our model is that all decellularized aortic valve conduits were not functional due to massive sinus thrombus formation. This was also found in the cellular allografts that became acellular. In the control cellular synergrafts, however, thrombus formation was not observed. Previous reports on decellularized valves are contradictory. Decellularized aortic valves transplanted into the pulmonary artery of the $\operatorname{dog}^{8}$ and porcine valves into the right outflow tract of a sheep heart ${ }^{15}$ did not show thrombus formation, although cryopreserved rat aortic valves with almost no endothelial cells have a high incidence of thrombus formation in the sinuses. ${ }^{26}$ An explanation could be that we used an heterotopic abdominal implantation in contrast 
to orthotopic transplantations in other studies. Due to the end-to-side implantation technique no physiological blood flow profile could be obtained, which can result in thrombus formation. The loss of endothelium, however, appeared to be an essential condition in thrombus formation.

In both the acellular allograft and synergraft valve leaflets ingrowth of transanastomotic intimal cells occurred. In one acellular synergraft almost three quarters of the valve leaflet was repopulated with SMC actin-positive cells. These SMC actin-positive cells were capable of producing the ECM molecules CS and FN. Interestingly, the loss of CS and FN, which was observed after the decellularization process, could be replenished by these cells. In the leaflets that had little ingrowth of intimal proliferation cells no replenishment of CS was seen. This unintended ingrowth of myofibroblasts indicates that the scaffold is suitable for repopulation and repair by autologous cells.

Although this study addressed the use of rat aortic valve conduit allografts for tissue-engineering research, the availability of human allograft valves is still limited. Tissue engineering of aortic valve xenografts could be a possible solution in overcoming this problem, due to the unlimited supply of porcine valves. The first tissue-engineered porcine heart valve implantation trials in humans have already been undertaken. ${ }^{15,27}$ However, decellularized arterial xenograft transplantations from guinea pig to rat abdominal aorta resulted in elastin degradation and aneurysmal dilatation, most probably due to an interspecies immunogenicity of the ECM. ${ }^{13}$ Although the first signs of these clinical trials are promising, the future will show us whether transplanted decellularized porcine valves have a better or worse prognosis than the generally used glutaraldehyde-fixed porcine valves. Meanwhile, further research is necessary to study the possible effects of immune response to interspecies ECM and to optimize decellularization techniques for human aortic valves.

Jan Lens is kindly acknowledged for his help in preparing the figures. The technical assistance of Maria Borrias and Conny van Munsteren is greatly appreciated.

\section{References}

1. Ross DN. Evolution of the homograft valve. Ann Thorac Surg. 1995; 59:565-7.

2. Clarke DR, Campbell DN, Hayward AR, Bishop DA. Degeneration of aortic valve allografts in young recipients. J Thorac Cardiovasc Surg. 1993;105:934-41.

3. Hogan P, Duplock L, Green M, et al. Human aortic valve allografts elicit a donor-specific immune response. J Thorac Cardiovasc Surg. 1996;112:1260-6.

4. Hoekstra FME, Knoop CJ, Vaessen LMB, et al. Donor-specific cellular immune response against human cardiac valve allografts. $J$ Thorac Cardiovasc Surg. 1996;112:281-6.

5. Green MK, Walsh MD, Dare A, et al. Histologic and immunohisto- chemical responses after aortic valve allografts in the rat. Ann Thorac Surg. 1998;66(Suppl):S216-20.

6. Moustapha A, Ross DB, Bittira B, et al. Aortic valve grafts in the rat: evidence for rejection. J Thorac Cardiovasc Surg. 1997;114:891-902.

7. Oei FBS, Welters MJP, Bonthuis F, et al. A size-matching heterotopic aortic valve implantation model in the rat. J Surg Res. 1999;87:239-44.

8. Wilson GJ, Courtman DW, Klement P, Lee JM, Yeger H. Acellular matrix: a biomaterials approach for coronary artery bypass and heart valve replacement. Ann Thorac Surg. 1995;60(Suppl):S353-8.

9. Bader A, Schilling T, Teebken OE, et al. Tissue engineering of heart valves-human endothelial cell seeding of detergent acellularized porcine valves. Eur J Cardiothorac Surg. 1998;14:279-84.

10. Steinhoff G, Stock U, Karim N, et al. Tissue engineering of pulmonary heart valves on allogenic acellular matrix conduits: in vivo restoration of valve tissue. Circulation. 2000;102(Suppl 3):III50-5.

11. Booth C, Korossis SA, Wilcox HE, et al. Tissue engineering of cardiac valve prostheses I: development and histological characterization of an acellular porcine scaffold. J Heart Valve Dis. 2002;11:457-62.

12. Bader A, Schilling TF, Teebken OE, et al. Tissue engineering of heart valves - human endothelial cell seeding of detergent acellularized porcine valves. Eur J Cardiothorac Surg. 1998;14:279-84.

13. Allaire E, Guettier C, Bruneval P, Plissonnier D, Michel JB. Cell-free arterial grafts: morphologic characteristics of aortic isografts, allografts, and xenografts in rats. J Vasc Surg. 1994;19:446-56.

14. Cebotari S, Mertsching H, Kallenbach K, et al. Construction of autologous human heart valves based on an acellular allograft matrix. Circulation. 2002;106:163-8.

15. Goldstein S, Clarke DR, Walsh SP, Black KS, O'Brien MF. Transpecies heart valve transplant: advanced studies of a bioengineered xenoautograft. Ann Thorac Surg. 2000;70:1962-9.

16. Dohmen PM, Lembcke A, Hotz H, Kivelitz D, Konertz WF. Ross operation with a tissue-engineered heart valve. Ann Thorac Surg. 2002;74:1438-42.

17. Legare JF, Lee TD, Creaser K, Ross DB. T lymphocytes mediate leaflet destruction and allograft aortic valve failure in rats. Ann Thorac Surg. 2000;70:1238-45.

18. Koolbergen DR, Hazekamp MG, Kurvers M, et al. Tissue chimerism in human cryopreserved homograft valve explants demonstrated by in situ hybridization. Ann Thorac Surg. 1998;66:s225-32.

19. Koolbergen DR, Hazekamp MG, de Heer E, et al. The pathology of fresh and cryopreserved homograft heart valves: an analysis of forty explanted homograft valves. J Thorac Cardiovasc Surg. 2002;124: 689-97.

20. Rajani B, Mee RB, Ratliff NB. Evidence for rejection of homograft cardiac valves in infants. J Thorac Cardiovasc Surg. 1998;115:111-7.

21. Salomon RN, Friedman GB, Callow AD, Payne DD, Libby P. Cryopreserved aortic homografts contain viable smooth muscle cells capable of expressing transplantation antigens. J Thorac Cardiovasc Surg. 1993;106:1173-80.

22. Hoekstra F, Witvliet M, Knoop C, et al. Donor-specific anti-human leukocyte antigen class I antibodies after implantation of cardiac valve allografts. J Heart Lung Transplant. 1997;16:570-2.

23. Oei FBS, Welters MJ, Vaessen LM, Stegmann AP, Bogers AJ, Weimar W. Induction of cytotoxic T lymphocytes with destructive potential after cardiac valve homograft implantation. J Heart Valve Dis. 2000;9:761-8.

24. Hilbert SL, Luna RE, Zhang J, et al. Allograft heart valves: the role of apoptosis-mediated cell loss. J Thorac Cardiovasc Surg. 1999;117: 454-62.

25. Oei FB, Welters MJ, Vaessen LM, et al. Heart valve dysfunction resulting from cellular rejection in a novel heterotopic transplantation rat model. Transpl Int. 2000;13(Suppl 1):S528-31.

26. Oei FB, Stegmann AP, Vaessen LM, Marquet RL, Weimar W, Bogers AJ. Immunological aspects of fresh and cryopreserved aortic valve transplantation in rats. Ann Thorac Surg. 2001;71(Suppl):S379-84.

27. Dohmen PM, Ozaki S, Verbeken E, Yperman J, Flameng W, Konertz WF. Tissue engineering of an auto-xenograft pulmonary heart valve. Asian Cardiovasc Thorac Ann. 2002;10:25-30. 